\title{
Children with Developmental Disability and Visual Impairment: What Information Can an Ophthalmologist Provide to Family and School
}

\author{
Sharon S. Lehman
}

Published online: 11 September 2013

(C) Springer Science + Business Media New York 2013

\begin{abstract}
Improved technology has increased survival of infants with serious medical conditions in the US, as well as worldwide, at a cost of significant developmental disability and sensory deficits in many children. This has created a public health challenge and is stressful to families who are the primary caretakers of children with complex medical problems resulting in disabilities. Children with comorbidities such as developmental disability and visual impairment have more difficulty than children with normal development. The pediatric ophthalmologist is an essential member of the multidisciplinary team caring for the child. The pediatric ophthalmologist can provide essential information and specific recommendations for medically necessary services, accommodations and interventions that can aid a child and family in reaching the child's potential and relieve stress.
\end{abstract}

Keywords Developmental disability .

Visual impairment · Ophthalmologist · Multidisciplinary team $\cdot$ Intervention $\cdot$ Accommodations $\cdot$ Services

\section{Introduction}

Although improved medical care has increased survival, significant concomitant disabilities remain a serious

S. S. Lehman $(\bowtie)$

Robison D. Harley MD Endowed Chair in Pediatric

Ophthalmology, Division of Ophthalmology, Department of

Surgery, Nemours Children's Clinic/AI duPont Hospital for

Children, 1600 Rockland Road, Wilmington, DE 19803, USA

e-mail: slehman@nemours.org

S. S. Lehman

Jefferson Medical College, Philadelphia, PA, USA

S. S. Lehman

Wills Eye Hospital, Philadelphia, PA, USA problem worldwide [1]. Manikin and colleagues performed a systematic review estimating risk of neurocognitive sequelae from perinatal insults. The deficits included cognition or learning, cerebral palsy and sensory deficits (hearing and vision). Approximately one-third of the children had multiple disabilities [2•].

In the US, prematurity remains a major cause of longterm morbidity for surviving children involving intellectual disability, behavior and overall health [3]. Specific recommendations for postnatal intervention may aid in helping involved infants reach their potential as well as limit family stress.

Significant levels of stress are reported in parents of children with developmental delay when compared with parents of children with normal development [4]. Behavioral, personal and social skill deficits are specific predictors of family stress. Specific recommendations for strategies for interventions and accommodations in these areas are important [5].

\section{Special Challenges Associated with Multiple Disability} and Visual Impairment

The combination of visual impairment and developmental disability is associated with more emotional and behavioral problems than in children with normal development [6•].

It may be difficult to diagnose visual impairment in children with additional disabilities. Expressive delay, behavioral issues and intellectual impairment may make interpretation of subjective responses to testing difficult. Welinder and Baggesen [7••] demonstrated visual impairment is common in children with severe developmental delay. Approximately $38 \%$ of children studied had visual impairment that was undiagnosed. It is important to include sensory testing in the 
workup and screening of children with developmental disability in the medical home as per American Academy of Pediatrics recommendations. If a child cannot be adequately screened, he or she should be referred to an eyecare specialist experienced in the care of children [8].

Children with autism can present a special challenge. Atypical visual behaviors associated with odd viewing angles and visual attention are seen in both autism and cortical visual impairment. Research involving neuroimaging and behavioral evidence suggests abnormalities in wiring and connections between specific areas of the brain are associated with the behaviors in autism [9, 10]. It is well known that cortical visual impairment involves abnormalities in ventral and dorsal brain visual processing pathways [11]. Both autism and cortical visual impairment are commonly seen in surviving premature infants $[12,13 \bullet \bullet]$. Care should be taken in assigning a diagnosis of autism based on atypical visual behaviors, especially by those unfamiliar with evaluating children with multiple disabilities. The totality of the evaluations and determinations of experienced members of the multidisciplinary team need to be looked at when this diagnosis is considered. The comorbidities may make assessment and diagnosis even more difficult. Whether performance is affected by visual impairment in cortical visual impairment or behavioral and perceptual responses in autism, accommodations and compensations can be based on concrete activities that are necessary in order to make progress in the educational environment.

\section{Parental Expectations}

Considering parents part of the multidisciplinary team for a child is essential to family-centered care. It is a widely accepted philosophy for a model of care in pediatrics and especially in the care of children with disabilities [14].

Jansen and colleagues studied parental expectations of medical care given to children with profound disabilities. Parents found "respectful and supportive care" and "enabling partnership" as most important. Parents of children in the age range 6-12 years of age were concerned that their need for "providing specific information about the child" was not being met [15•*]. Parents and society expect school age children to make educational progress. This expectation may fuel the desire for specific information. Specific recommendations for compensations and accommodations may aid the child in making progress in the classroom.

\section{Role of the Pediatric Ophthalmologist}

The pediatric ophthalmologist is an important member of the multidisciplinary team caring for a child with developmental disability and visual impairment in both the initial screening and ongoing evaluations.

\section{Visual Acuity Assessment}

The determination of visual acuity allows for vision services to be obtained for eligible children. Determination of visual acuity also aids others working with the child to supply materials that are appropriate in the educational, social and activities of daily living environments.

Preverbal and nonverbal children present special challenges. Common letter/picture acuity systems include the Snellen, HOTV, Allen and Lea symbols. If a typical letter or picture recognition acuity cannot be obtained, other methods may be employed. Preferential looking with contrast acuity grating cards or paddles is useful in this population. Repeat testing may be helpful. A temporal graph allows assessment over time and may be more valuable than any specific point in time that may have given poor results when a child's ability to cooperate may have been limited.

Some practitioners may prefer to estimate visual acuity with sweep visual evoked potentials. Studies show that this technique may be valuable in assessing visual acuity in nonverbal children [16].

Ophthalmologists may feel uncomfortable with giving specific information about a child's visual acuity because of a lack of familiarity with determination of acuity in this population or a lack of familiarity with alternative methods such as preferential looking or visual evoked potentials. It is important, however, to give some type of assessment of visual acuity. Morales and colleagues conducted visual acuity assessment using the preferential looking technique in a population of children with developmental disability and visual impairment referred by a pediatric ophthalmologist. They found that parental stress was reduced when a specific visual acuity was obtained and shared with the family. Pediatric ophthalmologists reported a quantitative visual acuity assessment aided in making a diagnosis and allowed for specific recommendations to be given to caretakers, educational institutions and early intervention programs $[17 \bullet \bullet]$.

Not all providers have experience with or access to such techniques as preferential looking techniques. The typical default is to use "central, steady and maintained" or "fix and follow" descriptions. Although these descriptions are helpful, a functional description may be of added value. An example of this type of description is: maintained fixation on 2 inch toy in midline and followed approximately 30 degrees off midline in either direction with both eyes open. Another example is: responds to moving lighted toy in right superotemporal field with both eyes open. This type of description aids family, caretakers, educational services 
and therapists in giving a starting place to visually engage the child. An additional comment about whether vision is consistent with or below what it expected for the child's age may help document disability. It is important to document whether your clinical impression meets the definition of the criteria for vision services in the state where the child lives and will receive services.

\section{Diagnosis}

Although some states base their eligibility for vision services on acuity alone, some base it on an ongoing ocular medical diagnosis that will impair the child's ability to progress in education and/or activities of daily living. It is imperative that children receive a diagnosis causing their visual impairment. A commonly overlooked diagnosis that is not mentioned in reports is cortical visual impairment in children with profound multiple disabilities (e.g., quadriplegic cerebral palsy). The absence of this diagnosis may limit a child's ability to obtain vision services.

Educational materials including disease course and prognosis, if applicable, should be made available to the family and other relevant members of the multidisciplinary team. Other team members may be copied via the electronic health record or the information may be given to the family who may distribute it to members of the team based on their desire to share the information. Information about relevant websites and support groups is helpful. It is important to have printed material available since not all patients and families have Internet access.

It is important to discuss genuine current research and clinical trials. Sham treatments are present on the Internet, and desperate families may be vulnerable to the empty promises that may be made. This information about genuine research also gives families hope that there may be progress made concerning treatment during their child's lifetime.

\section{Specific Recommendations for Patient, Family} and Others

It is important that the ophthalmologist documents any evaluations that are needed and accommodations that are medically necessary. This allows for caretakers and educational services to incorporate these items into the child's day. It will be incorporated into an IEP (Individual Educational Plan) if the child has one. Not all children will have quality advocates or services depending upon the geographic location, etc. It is important that the ophthalmologist consults with the family and other multidisciplinary team members and documents all medically necessary accommodations.

Not all ophthalmologists are experienced in providing these recommendations. There are several strategies that may help in constructing the recommendations that should be transmitted in print for families to ensure that their child is receiving the proper services and accommodations. The electronic health record allows for the construction of practical and efficient transmission of this information to the family and other members of the multidisciplinary team.

The first schema for specific recommendations is based on diagnosis. Certain clinical signs and symptoms may be associated with a diagnosis. What is expected based on the diagnosis is evaluated and recommendations made based on the patient's history and examination. A patient with optic atrophy from optic chiasmal involvement of a glioma may be expected to have decreased acuity, abnormal color vision, poor contrast acuity and visual field defects. An example of a list of recommendations based on diagnosis is listed in Table 1.
Table 1 Specific

recommendations for middle school student with a diagnosis of visual impairment from optic atrophy secondary to chiasmal lesion

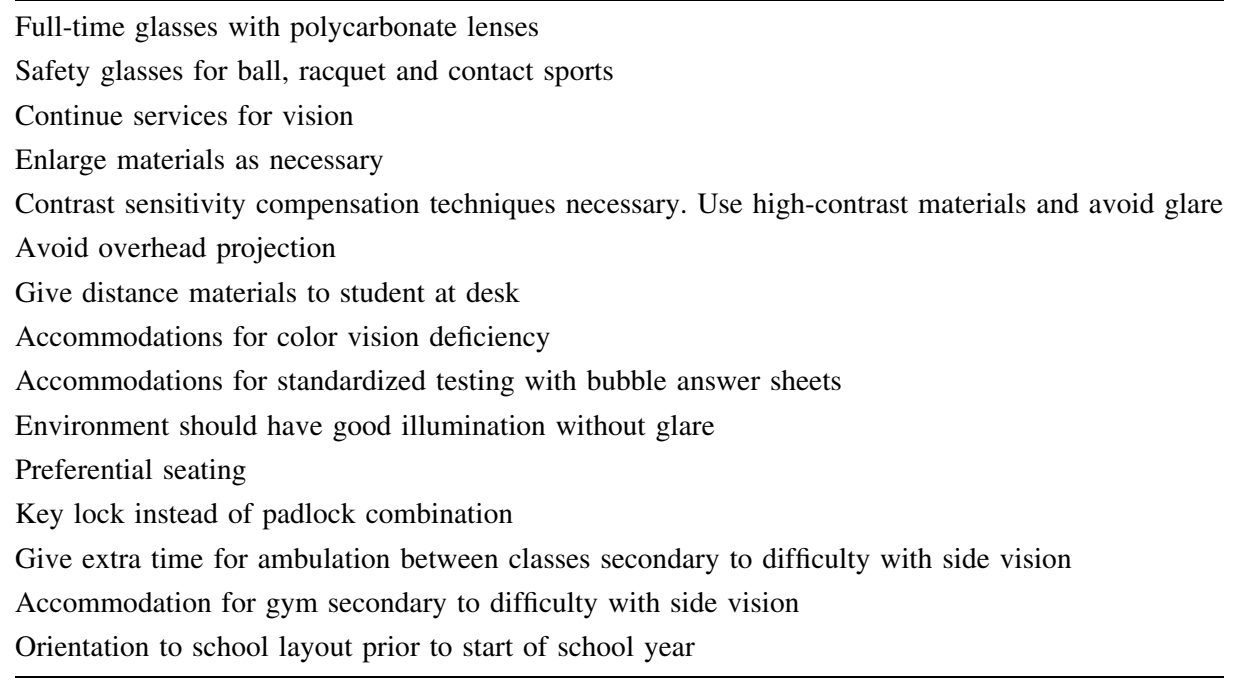

Safety glasses for ball, racquet and contact sports

Continue services for vision

Enlarge materials as necessary

Contrast sensitivity compensation techniques necessary. Use high-contrast materials and avoid glare

Avoid overhead projection

Give distance materials to student at desk

Accommodations for standardized testing with bubble answer sheets

Environment should have good illumination without glare

Preferential seating

Key lock instead of padlock combination

Give extra time for ambulation between classes secondary to difficulty with side vision

Accommodation for gym secondary to difficulty with side vision 
Table 2 Checklist of categories to be used as tool to develop specific recommendations for children with developmental delay and visual impairment

Environment (e.g., room illumination, glare, avoid clutter, etc.)

Characteristics of visual materials (e.g., high contrast print, magnification, etc.)

Activities of daily living (e.g., enlarged computer screen and keyboard, audiobooks, etc.)

Methods of communication (e.g., switches, augmentative devices, etc.)

Orientation and mobility (e.g., evaluation for services, service animal, etc.)

Another schema for specific recommendations involves using a checklist of categories to be reviewed with the family and other members of the multidisciplinary team and tailoring the recommendations to the problems the patient is encountering. The categories include: environment, visual materials, activities of daily living (tasks involved in progressing through one's day), communication and orientation and mobility (Table 2).

The final schema is basing specific recommendations on an evaluation tool that documents the presence of specific criteria. Roman-Lantzy has developed a tool, the cortical visual impairment characteristic assessment method, which can be used to plan for specific recommendations, accommodations and interventions based on the presence or resolution of traits that are essential to the diagnosis of cortical visual impairment [18]. It is a complete and lengthy tool used often by teachers of the visually impaired or occupational therapists that provide vision services. Communication of these assessments should be shared with the entire team caring for the child.

\section{Conclusions}

The pediatric ophthalmologist can provide essential information as part of the multidisciplinary team caring for children with developmental disability and visual impairment. The pediatric ophthalmologist is uniquely qualified to assess visual acuity and make a diagnosis in a child with complex medical conditions. Both documentation of acuity and diagnosis are essential for children to qualify for medically necessary services, accommodations and interventions. Because of the importance of this role, it behooves the pediatric ophthalmologist to become comfortable and familiar with caring for the child with multiple disabilities and meeting their needs, including constructing specific recommendations that impact the child's educational and behavioral progress. Communication is key. Evaluations from members of the multidisciplinary team caring for a child should be shared with the family and others on the team. This information can lessen the stress of both child and family, thus impacting their well-being.

\section{Compliance with Ethical Requirements}

Conflict of interest Sharon S. Lehman declares that she has no conflict of interest.

Human and Animal Rights and Informed Consent This article does not contain any studies with human or animal subjects performed by any of the authors.

\section{References}

Papers of particular interest, published recently, have been highlighted as:

- Of importance

•- Of major importance

1. van Sorge AJ, Termote JUM, de Vries MJ, Boonstra FN, Stellingwerf C, Schaly-Delfos NE. The incidence of visual impairment due to retinopathy of prematurity (ROP) and concomitant disability in the Netherlands: a 30 year overview. Br J Ophthalmol. 2011;95:937-41.

2. - Mwaniki MK, Atieno M, Lawson JE, Newton CRJ. Long-term neurodevelopmental outcomes after intrauterine and neonatal insults: a systematic review. Lancet 2012;379:445-452. Study describes the incidence of children with multiple disabilities.

3. McCormick MC, Litt JS, Smith VC, Zupancic JAF. Prematurity: an overview and public health implications. Annu Rev Public Health. 2011;32:367-79.

4. Estes A, Munson J, Dawson G, Kochler E, Zhou XH, Abbott R. Parenting stress and psychological functioning among mothers of preschool children with autism and developmental delay. Autism. 2009;13(4):375-87.

5. Webster RI, Majnemer A, Platt RW, Shevell MI. Child health and parental stress in school-age children with a preschool diagnosis of developmental delay. J Child Neurol. 2008;23:32-8.

6. Alimovic S. Emotional and behavioural problems in children with visual impairment, intellectual and multiple disabilities. J Intellectual Disabil Res. 2013;57:153-160. Helps healthcare providers understand patient and family challenges.

7. •• Welinder LG, Baggesen KL. Visual abilities of students with severe developmental delay in special needs education-a vision screening project in Northern Jutland, Denmark. Acta Ophthalmol. 2012;90:721-726. Reminds us that visual loss in children with developmental disability can be overlooked.

8. Committee on Practice and Ambulatory Medicine, Section of Ophthalmology, American Association of Certified Orthoptists, American Association of Pediatric Ophthalmology and Strabismus, American Academy of Ophthalmology. Eye examination in infants, children and young adults by pediatricians. Pediatrics. 2003;111(4):902-7.

9. Brenner LA, Turner KC, Muller RA. Eye movement and visual search: Are there elementary abnormalities in autism? J Autism Dev Disord. 2007;37:1289-309.

10. Johnson BP, Rinehart NJ, Papadopoulos N, Tonge B, Millist L, White O, Fielding J. A closer look at visually guided saccades in autism and Asperger's disorder. Front Intergrative Neurosci. 2012;6:1-7.

11. Goodale MA, Milner AD. Separate visual pathways for perception and action. Trends Neurosci. 1992;1(1):20-5. 
12. Stephens BE, Bann CM, Watson VE, et al. Screening for autism disorders in extremely preterm infants. J Dev Behav Pediatr. 2012;33:535-54.

13. • Siatkowski RM, Good WV, Summers CG, Quinn GE, Tung B. Clinical characteristics of children with severe visual impairment but favorable retinal structural outcomes from the Early Treatment for Retinopathy of Prematurity (ETROP) study. J Am Assoc Pediatr Ophthalmol Strabismus. 2013;17(2):129-134. Welldesigned, long-term review of vision loss in former premature infants not due to abnormal retinal structure.

14. Duby JC, American Academy of Pediatrics Council on Children with Disabilities. Role of the medical home in family-centered early intervention services. Pediatrics. 2007;120(5):1153-8.

15. • Jansen SLG, van der Patten AAJ, Vlaskamp C. What parents find important in the support of a child with profound intellectual and multiple disabilities. Child: Care Health Dev. 2012;39(3): 432-441. Important article that informs healthcare providers about parental expectations.

16. Almoqbel F, Leat SJ, Irving E. The technique validity and clinical use of the sweep VEP. Ophthalmic Physiol Opt. 2008;28: 393-403.

17. $\bullet$ Morale SE, Hughbrooks-Wheaton DK, Cheng C, Subramanian V, O'Connor AR, Birch EE. Visual acuity assessment of children with special needs. Am Orthopt J. 2012;62:90-98. Advantages of obtaining preferential looking visual acuity testing in children with developmental disability.

18. Roman-Lantzy C. Cortical visual impairment: an approach to assessment and intervention. New York: AFB Press; 2007. 